\title{
Spontaneous Remission of Sick Sinus Syndrome in a Fetus with Pulmonary Stenosis Regurgitation
}

\author{
Kazuhiro Kajiwara, MD, $\mathrm{PhD}^{1} \quad$ Satoru Ishikawa, $\mathrm{MD}^{2} \quad$ Takuma Mori, MD $\quad$ Osamu Samura, MD, $\mathrm{PhD}^{1}$ \\ Aikou Okamoto, MD, $\mathrm{PhD}^{1}$ \\ ${ }^{1}$ Department of Obstetrics and Gynecology, The Jikei University \\ School of Medicine, Tokyo, Japan \\ 2 Department of Pediatrics, The Jikei University School of Medicine, \\ Tokyo, Japan

\begin{abstract}
Address for correspondence Kazuhiro Kajiwara, MD, PhD, Department of Obstetrics and Gynecology, The Jikei University School of Medicine, Tokyo 105-8471, Japan (e-mail: Kajiwarakaiki.db@gmail.com).
\end{abstract} \\ Am J Perinatol Rep 2019;9:e372-e375.
}

\begin{abstract}
Keywords

- pulmonary stenosis regurgitation

- sick sinus syndrome

- fetal echocardiography

- fetal bradyarrhythmia

- congenital heart disease pregnancy progresses.

Congenital sick sinus syndrome (SSS) is characterized by dysfunction of the sinoatrial node which results in inappropriate sinus bradycardia, sinus arrest, or chronotropic incompetence. Approximately $80 \%$ children with SSS have a history of cardiac surgery for congenital heart disease (CHD), ${ }^{1-4}$ and SSS is also observed in fetuses with CHD. ${ }^{5}$ However, detailed mechanisms underlying the development of SSS are not fully understood. Although the incidence of fetal SSS is not fully understood, it accounts for approximately $50 \%$ of cases of bradycardia with CHD and $9 \%$ of cases of bradycardia without CHD. ${ }^{5}$ Tricuspid regurgitation might cause SSS, but there is no report about the relationship between SSS and PSR during pregnancy, particularly in spontaneously resolved cases. Here we report a case of fetal SSS caused by pulmonary stenosis regurgitation (PSR) that spontaneously resolved during pregnancy.
\end{abstract}

Objective Here, we report a case of fetal sick sinus syndrome (SSS) caused by pulmonary stenosis regurgitation (PSR) that spontaneously resolved during pregnancy. Case Report A 29-year-old woman was referred to our hospital at 21 weeks of gestation for persistent fetal bradycardia. Fetal echocardiography revealed PSR and ventricular septal defect (VSD). The ventricular rate was 60 to 70 beats/minute with 1:1 atrioventricular conduction. Thus, congenital SSS owing to PSR was suspected. During pregnancy, fetal SSS spontaneously resolved at 28 weeks of gestation despite persistent PSR. The ventricular rate was increased to approximately 120 beats/minute with regular rhythm. A 2,390-g male neonate was delivered via Caesarean section at 38 weeks of gestation. Consequently, detailed echocardiography revealed PSR and VSD without SSS.

Conclusion Although fetal PSR can cause fetal SSS owing to immaturity at an earlier gestational age, SSS might be spontaneously resolved by fetal heart development as

\section{Case Description}

A 29-year-old woman, gravida 1, para 0, was referred to our hospital at 21 weeks of gestation because of persistent fetal bradycardia. She did not have any history of familial genetic disease. A detailed ultrasonographic examination (Voluson E8 GE Healthcare, Milwaukee, WI) revealed sinus bradycardia (60-70 beats/minute $[\mathrm{bpm}]$ ) with $1: 1$ atrioventricular (AV) conduction (-Fig. 1A, b). The four-chamber view showed ventricular septal defect (VSD; - Fig. 2). The diameter of VSD was $4 \mathrm{~mm}$. The size of ventricles and atria were proportionate and did not show enlargement. The three-vessel view showed an enlarged main pulmonary artery. The right outflow tract showed pulmonary valve stenosis with regurgitation ( - Fig. 3A, B). No blocked atrial extrasystoles or tachyarrhythmia was detected, and maternal autoantibodies were negative; thus, congenital SSS owing to PSR was suspected. The fetal status was good without any sign of hydrops. Examinations at 24 and 26 weeks of gestation revealed sustained fetal bradycardia. However, fetal bradycardia spontaneously resolved at 28 weeks of gestation without any fetal treatment, regardless of persistent PSR (-Fig. 4). The ventricular rate was approximately $120 \mathrm{bpm}$. Thereafter, although the degree of PSR did not change in the fetus, SSS was absent throughout the pregnancy received

July 10, 2019

accepted

July 12, 2019
DOI https://doi.org/

10.1055/s-0039-1695745. ISSN 2157-6998.
Copyright $\odot 2019$ by Thieme Medical

Publishers, Inc., 333 Seventh Avenue, New York, NY 10001, USA. Tel: +1(212) 584-4662.
License terms

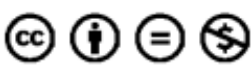




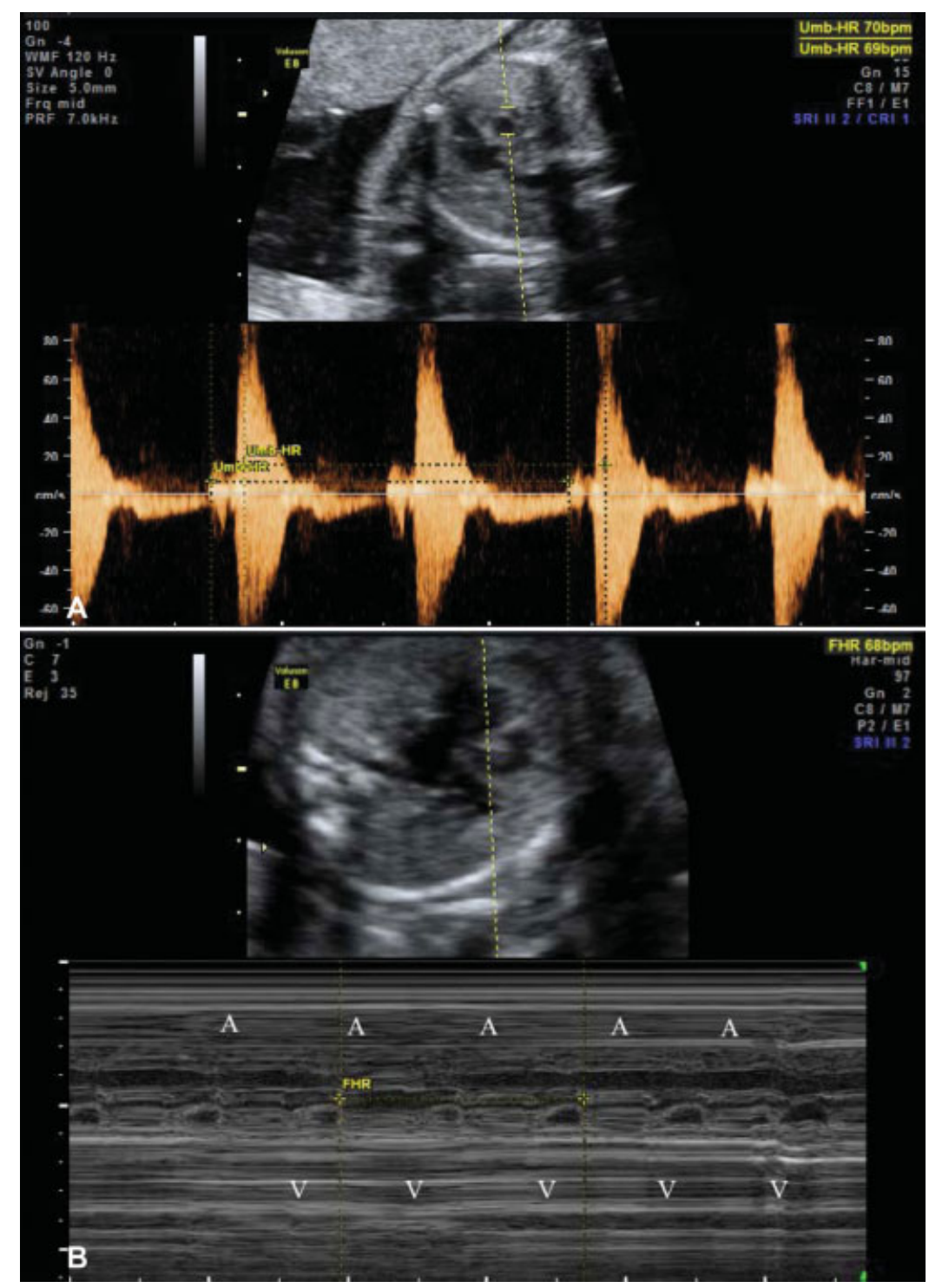

Fig. 1 Prenatal diagnosis of sick sinus syndrome and pulmonary stenosis regurgitation. (A) Simultaneous pulsed Doppler interrogation of flow in the superior vena cava and ascending aorta showed sustained fetal bradycardia with regular rhythm and 1:1 atrioventricular conduction.

(B) M-mode assessment with the cursor placed through the right atrium (RA) and left ventricle (LV) showed 1:1 atrioventricular conduction. The ventricular rate was $68 \mathrm{bpm}$. Apical four-chamber view showing ventricular septal defect. bpm, beats/minute.

and there were no signs of cardiac failure. A male neonate weighing 2,390 $\mathrm{g}$ was delivered via Cesarean section at 38 weeks of gestation with an Apgar's score of 8 at both 1 and 5 minutes. Consequently, detailed echocardiography revealed PSR and VSD with regular heart rhythm. The results of initial electrocardiography were normal. Thus, the postnatal diagnosis was consistent with prenatal impressions obtained. The neonate was discharged with an uneventful observation 27 days after birth. During the last hospital visit, the 8-monthold infant had PSR without any signs of congestive heart failure and was a relatively slow growth rate and 3-month developmental retardation. His heart rate was approximately $110 \mathrm{bpm}$ with regular heart rhythm.

\section{Discussion}

Fetal bradycardia is defined as a fetal heart rate with a baseline of $<110 \mathrm{bpm}$. The major causes of bradycardia include con- genital SSS, AV block, long-QT syndrome and nonconducted atrial premature beats. ${ }^{6}$ Sustained fetal bradycardia associated with regular and 1:1 AV conduction basically suggests SSS. Fetal bradycardia may be present in fetuses with and without CHD. In a fetus without CHD, antibodies, such as anti-Ro/ Sjogren's syndrome A antibodies, are often associated with fetal bradyarrhythmia owing to AV block. ${ }^{7}$ Conversely, in a fetus with CHD, congenital bradyarrhythmia is believed to be caused by the structural defect itself. ${ }^{8}$ The discontinuity between the AV node and ventricular conduction tissues that is often caused by heterotaxy and corrected transposition of the great arteries can result in SSS. Additionally, SSS can be caused by hemodynamic stress and aging. ${ }^{9,10}$ The severity of tricuspid regurgitation after the additive maze procedure during mitral valve surgery has been reported to be associated with SSS development. ${ }^{10}$ In the study, the late-onset SSS group had a higher mean age, suggesting that age has an impact on the cumulative risk of developing SSS. ${ }^{10}$ Noda et al reported 
e374 Spontaneous Remission of Sick Sinus Syndrome in a Fetus Kajiwara et al.

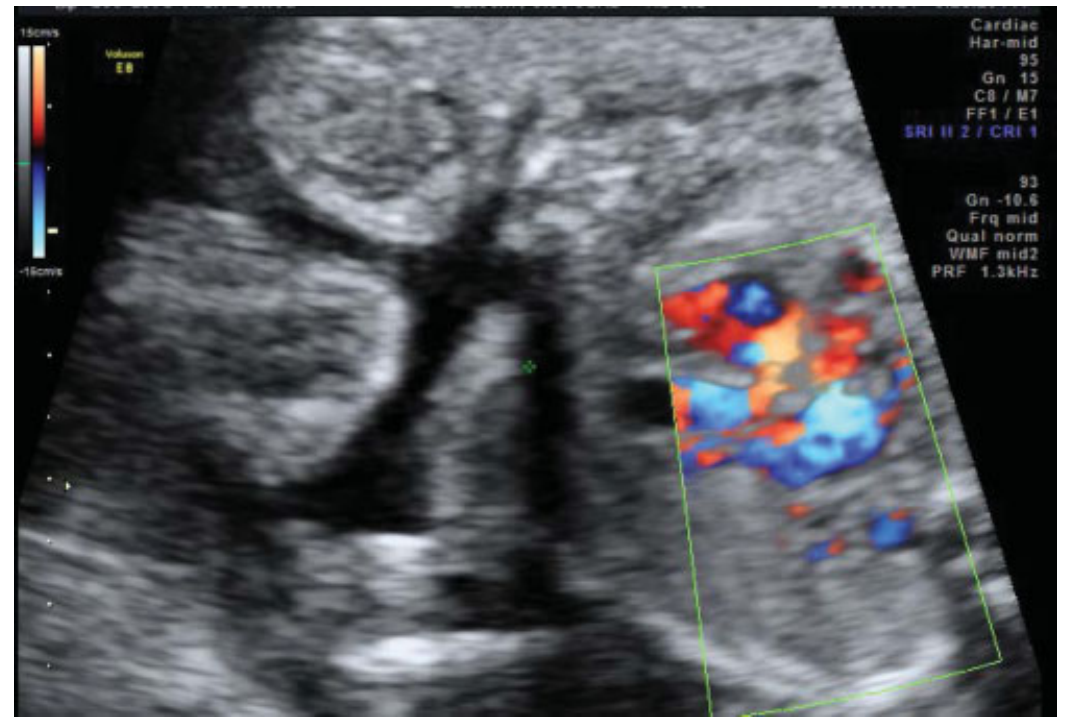

Fig. 2 Apical four-chamber view showing ventricular septal defect.
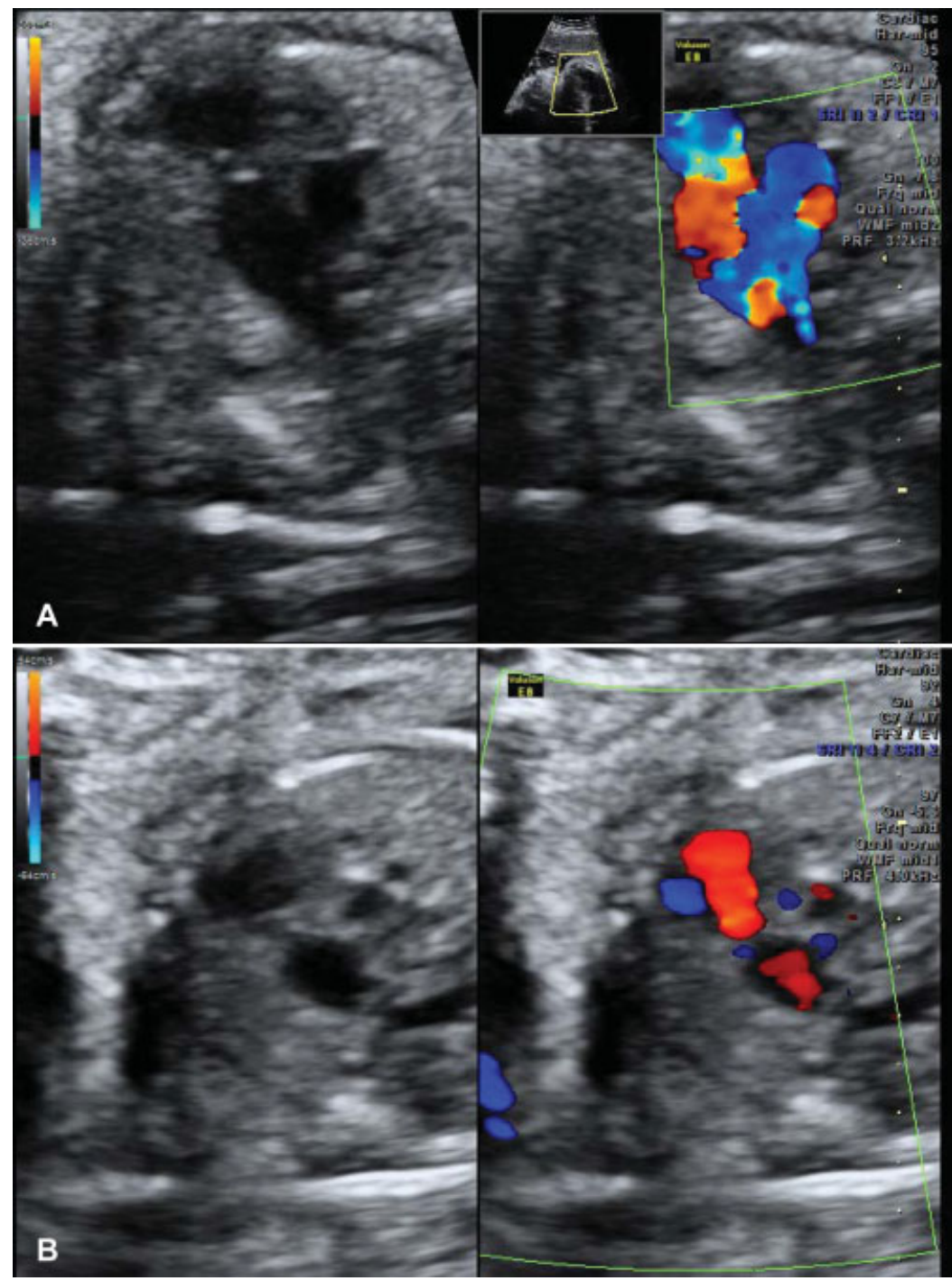

Fig. 3 Image showing right ventricular outflow tract (RVOT). RVOT demonstrated pulmonary valve stenosis (A) and pulmonary regurgitation (B). 


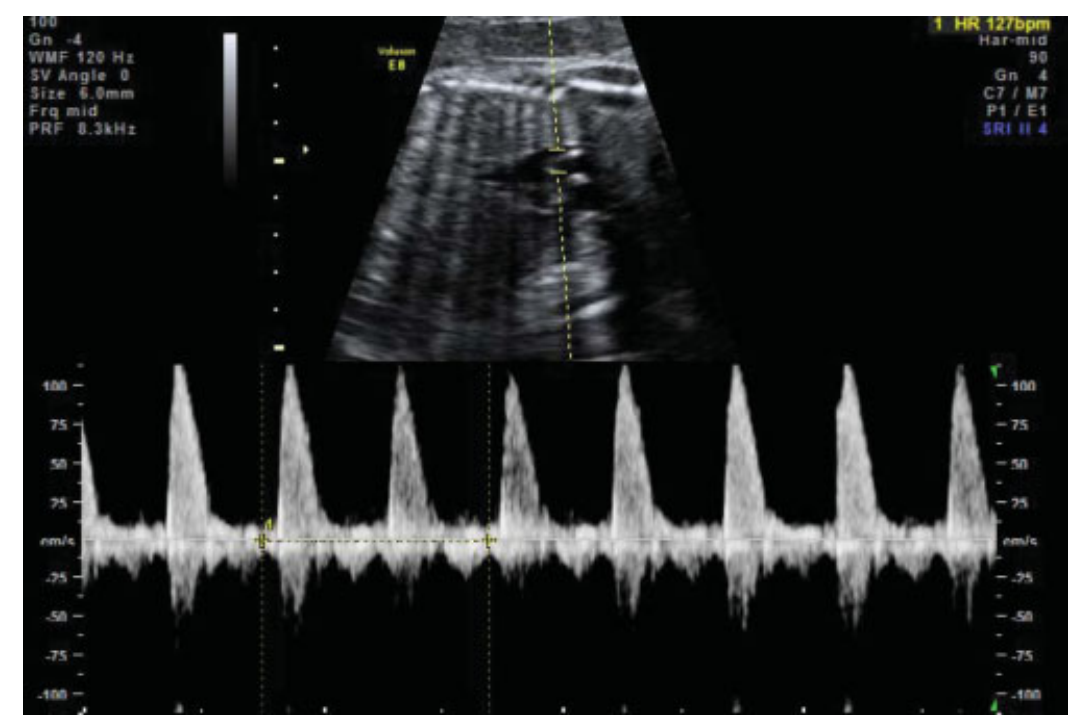

Fig. 4 Simultaneous pulsed Doppler interrogation of flow in the superior vena cava and ascending aorta at 28 weeks of gestation showed regular heart rhythm without fetal bradycardia. The ventricular rate was 127 bpm. bpm, beats/minute.

that tachyarrhythmia spontaneously changed to SSS and finally changed to complete heart block in patients with Ebstain's anomaly over a 16 -year period. ${ }^{11}$ The author indicated that cell damage in the heart's conduction system and accessory pathway may cause SSS even in a situation without hemodynamic stress because the patients were young and had almost normal hemodynamics. Taken together, SSS can be caused by not only hemodynamic stress but also age-related changes, such as apoptosis and fibrosis in cardiac cells, suggesting that the dramatic development of fetal cardiac cell may affect the condition of SSS.

Hemodynamic stress owing to pulmonary valve regurgitation of the sinoatrial node in the absence of fetal heart maturity might have caused SSS in our case. However, SSS was resolved by maturation of the fetal heart as pregnancy progressed despite persistent PSR. Our report indicates the possibility of spontaneous disappearance of SSS caused by CHD, particularly in the case of hemodynamic stress, such as regurgitation. It is important to mention this when counseling the mother so that she is aware of the actual fetal prognosis.

\section{Conclusion}

In conclusion, fetal PSR can cause SSS owing to fetal heart immaturity at an earlier gestational age. Consequently, fetal SSS can be resolved spontaneously as pregnancy progresses.

\section{Conflict of Interest}

None.

\section{References}

1 Yabek SM, Swensson RE, Jarmakani JM. Electrocardiographic recognition of sinus node dysfunction in children and young adults. Circulation 1977;56(02):235-239

2 Yabek SM, Jarmakani JM. Sinus node dysfunction in children, adolescents, and young adults. Pediatrics 1978;61(04):593-598

3 Radford DJ, Izukawa T. Sick sinus syndrome. Symptomatic cases in children. Arch Dis Child 1975;50(11):879-885

4 Kugler JD, Gillette PC, Mullins CE, McNamara DG. Sinoatrial conduction in children: an index of sinoatrial node function. Circulation 1979;59(06):1266-1276

5 Miyoshi T, Maeno Y, Sago H, et al. Fetal bradyarrhythmia associated with congenital heart defects - nationwide survey in Japan. Circ J 2015;79(04):854-861

6 Lin MT, Hsieh FJ, Shyu MK, Lee CN, Wang JK, Wu MH. Postnatal outcome of fetal bradycardia without significant cardiac abnormalities. Am Heart J 2004;147(03):540-544

7 Buyon JP, Ben-Chetrit E, Karp S, et al. Acquired congenital heart block. Pattern of maternal antibody response to biochemically defined antigens of the SSA/Ro-SSB/La system in neonatal lupus. J Clin Invest 1989;84(02):627-634

8 Ho SY, Fagg N, Anderson RH, Cook A, Allan L. Disposition of the atrioventricular conduction tissues in the heart with isomerism of the atrial appendages: its relation to congenital complete heart block. J Am Coll Cardiol 1992;20(04):904-910

9 Pexieder T. Cell death in the morphogenesis and teratogenesis of the heart. Adv Anat Embryol Cell Biol 1975;51(03):3-99

10 Cho MS, Heo R, Jin X, et al. Sick sinus syndrome after the maze procedure performed concomitantly with mitral valve surgery. J Am Heart Assoc 2018;7(19):e009629

11 Noda M, Yamaguchi H, Fujii H, et al. Spontaneous regression over a 16-year period of tachyarrhythmias to sick sinus syndrome and complete atrioventricular block in a young patient with Ebstein's anomaly. Pacing Clin Electrophysiol 2001;24(07):1158-1160 